Nowacka Agnieszka, Śniegocki Maciej, Woźniak-Dąbrowska Kamila, Smuczyński Wojciech. Microvessels in tumor tissue in patients with central nervous system tumors. Journal of Education, Health and Sport. 2021;11(02):16-29. eISSN 2391-8306. DOI http://dx.doi.org/10.12775/JEHS.2021.11.02.002

https://apcz.umk.pl/czasopisma/index.php/JEHS/article/view/JEHS.2021.11.02.002

https://zenodo.org/record/4527819

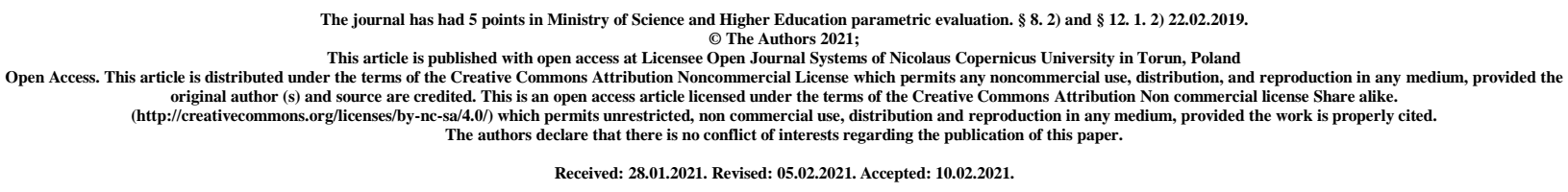

\title{
Microvessels in tumor tissue in patients with central nervous system
} tumors

\author{
Agnieszka Nowacka ${ }^{1}$, Maciej Śniegocki ${ }^{1}$, Kamila Woźniak-Dąbrowska ${ }^{1}$, \\ Wojciech Smuczyński \\ ${ }^{1}$ Department of Neurosurgery, Nicolaus Copernicus University Collegium Medicum in Bydgoszcz, \\ Poland \\ ${ }^{2}$ Department of Physiotherapy, Nicolaus Copernicus University Collegium Medicum in Bydgoszcz, \\ Poland
}

\begin{abstract}
Angiogenesis plays an essential role in the development of a tumor. The most preferred histological evaluation method of this process is the determination of microvessel density (MVD) in the biopsy. Numerous studies report the possibility of using various MRI techniques for imaging the network of neoplastic vessels and determining the angiogenesis in them, as well as the subsequent analysis of the obtained images. In this study, a classic MRI imaging with intravenous contrast agent administration was used, and in order to ensure objectification and repeatability, and to eliminate human error that may occur in classic MRI image analysis, an automatic evaluation algorithm was used to determine the degree of angiogenesis, which was then presented in the form of a statistical pixels analysis. The study involved 48 adult patients of both sexes, treated surgically at the Department of Neurosurgery, Nicolaus Copernicus University Collegium Medicum in Bydgoszcz, due to a solid brain tumor. The highest mean density of MVD microvessels in tumor tissue was observed in high-grade gliomas. The high level of MVD expression in tumors of the central nervous system showed a positive correlation with the high degree of tumor malignancy. Based on the conducted research, it was concluded that the determination of the level of MVD expression in the tumor tissue is an additional prognostic factor in neoplasms of the central nervous system. The comparison of the results obtained with the tests performed routinely translates into a more accurate selection of treatment and assessment of the prognosis for survival.
\end{abstract}

Key words: MVD; MRI, angiogenesis; brain tumors 


\section{Introduction}

Neoangiogenesis plays an essential role in the development of a neoplasm. Blood vessels determine its development and metastasis. It can therefore be concluded that the greater number of microvessels within the tumor correlates with its degree of malignancy (microvessel density - MVD - is considered a prognostic factor in many neoplasms).

In the case of histopathological examinations, the most recommended method of assessing neoangiogenesis is the definition of the so-called microvessel density (MVD) $[1,2]$ in the hot spots by expression of endothelial antigen CD105, CD34, von Willebrand factor [3] or fascin (CD31) by the light microscopy. In this study, immunohistochemical staining for CD34 was used to evaluate MVD in tumor tissue, using rabbit polyclonal antibodies to CD34 (Rabbit Polyclonal to CD34, ABCAM). Obtained data was correlated with the data obtained in the analysis of preoperative MRI images of brain tumors. Numerous studies report the possibility of using various MRI techniques for imaging the neoplastic vessel network and determining the angiogenesis in them [4] as well as the subsequent analysis of the obtained images [5,6]. In this study, the classic MRI imaging (sequences: T1, T2, T1 $+\mathrm{c}, \mathrm{T} 2+\mathrm{c}$ ) with intravenous administration of a contrast agent (gadolinium) was used. In order to ensure objectivity and repeatability, and to eliminate human error that may occur in classic MRI image analysis, an automatic evaluation algorithm was used to determine the degree of angiogenesis, which was then presented in the form of statistical analysis of pixels.

\section{Materials and methods}

In order to conduct the research, the approval of the Bioethics Committee of Nicolaus Copernicus University Collegium Medicum in Bydgoszcz was obtained, its number being: KB$665 / 2009$. The study involved 48 adult patients $\left(M_{b}=60.16\right)$ of both genders $(21$ women and 27 men), treated surgically for brain tumor in the Department of Neurosurgery, Nicolaus Copernicus University Collegium Medicum in Bydgoszcz. Qualification of patients was based on interview, physical as well as neuroimaging examinations, and in the later stage it was confirmed with the post-surgical histopathological examination of the removed tumor. All people who were qualified for the study gave their written consent to take part in it. Within the studied group, the youngest person was 26 years old, and the oldest one was 79 years old. The diversity of age was equal to over a decade ( $\mathrm{SD}=10.79)$. The majority of patients had grade III $(27.08 \%)$ or IV $(33.33 \%)$ tumors, according to WHO. Grade I and II was characteristic for $20.83 \%$ and $18.75 \%$ of patients, respectively.

The frequency of the occurrence of the particular tumor types was assessed by the division into the 5 general groups of intracranial tumors: high grade gliomas (HGG), low-grade gliomas (LGG), meningiomas, metastatic tumors and others (Antoni B type schwannoma, Antoni A type schwannoma and adenoma hypophysis). The majority of people of the studied group had gliomatype tumors - in total, they constituted $62.5 \%$ of the studied group. The type that prevailed the most often were HGG; they were found in almost half of the studied group (47.92\%). 14.58\% of the studied group had low-grade gliomas. The next type - meningiomas, was characteristic in every $6^{\text {th }}$ studied patient (18.75\%). Metastatic tumors were characteristic for $12.5 \%$ of patients. The tumors from the last group were found the least often - in slightly more than $6 \%$ of the studied group. 
The study in the researched group was conducted according to the following scheme:

1. Gaining written, informed patient's consent to take part in the study.

2. Performing an MRI of the head before the surgery and evaluation of the obtained image.

3. Performing the planned surgery consisting in removing the brain tumor.

4. Histological assessment of the material gained from the surgically removed brain tumor.

Pre-operative imaging of the brain tumor with intravenous (gadolinium) administration was performed according to the standard procedures of this study. The obtained MRI images were then assessed and analyzed to determine the degree of angiogenesis. For this purpose, a proprietary automatic evaluation algorithm was used, which was to ensure objectivity and repeatability, and to eliminate human error that may occur in the classic MRI image analysis.

The brain tumor samples gained during the surgery underwent histopathological examination. The specimens made with paraffin blocks were assessed as follows:

1. Determining the histological type of the tumor and its grade of malignancy.

2. Assessing MVD in tumor tissue, using immunohistochemical staining for CD34, using the following reagents: Rabbit Polyclonal to CD34, by ABCAM and EnVision ${ }^{\circledR}+$ Kits, HRP Rabbit (AEC+), by Dako.

The histological type of tumors and their malignancy grade were determined according to the WHO classification of tumors of the central nervous system. A detailed analysis was carried out on one slice of each tumor sample, that contained at least three angiogenic "hot-spots" (areas with the greatest density of microvessels on a tissue section), that were representative for the whole lesion. Then, the selected specimens were stained and checked in three "hot-spots" for the presence of VEGF-A, VEGFR-2 and MVD. The intensity of the immunohistochemical reaction was assessed by using light microscope, using the IRS scale according to Remmele, taking into account the intensity of staining and the proportion of the cells in which the reaction was observed.

The statistical analysis of the collected data was carried out using the Statistica 9.0 kit. Descriptive statistics and descriptive characteristics were used to describe the variables. For most of the tested variables, a deviation from the normal distribution was found, tested by Lillefors test ( $p$ $<0.01$ ), that's why nonparametric statistics were used. Spearman's correlation coefficient matrix was used to examine relations among variables. The Man-Whitney $U$ test was used examine the differences between the variables in the comparison of divalent variables, and to compare multivariant variables - Anova Kruskal-Wallis test. Comparison of the same variable between the two measurements was made using the Wilcoxon pair test. All results with the condition $<0.05$, were considered statistically significant.

\section{Results}

As a result of the analysis of the MRI images, statistics were obtained for the results computed for the whole images (all sections), using the mask (found by the BET program). Descriptive statistics (minimum, maximum, arithmetic mean, variance) were determined before and after contrast administration. The difference in distances between the histograms was calculated by two methods: Manhattan and Earth Mover's Distance. The first one calculates the sum of the absolute values of the differences between the individual bars of the histogram and resembles the classical mathematical Euclidean distances, the second one calculates the minimal cost of moving the bars of one of the histograms to obtain the other. The same values are calculated for the left and right image halves. 
In the next step, the results found without the mask were analyzed, but the pixels shining less than $5 \%$ of the maximum intensity were removed, in the next step - a mask was used. The first goal was to take advantage of the fact that the tumor is most often located asymmetrically, and the second was to overlay any scale on the distances between histograms by finding it between image halves before contrast. For the data prepared in this way, the mean descriptive statistics were determined, as presented in Table 1.

Table 1. Statistics for mean values from the analysis of MRI histograms (before and after contrast administration).

\begin{tabular}{|c|c|c|c|c|c|c|c|}
\hline & $\begin{array}{c}\text { N } \\
\text { important }\end{array}$ & Mean & Median & Min & Max & $\begin{array}{c}\text { Standard } \\
\text { deviation }\end{array}$ & $\begin{array}{c}\text { Coefficient } \\
\text { of variation }\end{array}$ \\
\hline Med. BC & 48 & 467,04 & 457,88 & 216,698 & 746,9 & 99,81 & 21,3713 \\
\hline Var. BC & 48 & 16715,63 & 15243,04 & 4950,292 & 57706,5 & 8230,39 & 49,2377 \\
\hline Med. AC & 48 & 534,42 & 525,81 & 116,759 & 1816,5 & 217,77 & 40,7496 \\
\hline Var. AC & 48 & 34301,59 & 22736,53 & 973,019 & 553136,6 & 77087,84 & 224,7355 \\
\hline $\begin{array}{c}\text { Manhattan } \\
\text { Earth Mover's }\end{array}$ & 48 & 0,55 & 0,44 & 0,208 & 1,9 & 0,32 & 58,9114 \\
\hline Distance & 48 & 20,78 & 14,21 & 6,800 & 169,4 & 30,72 & 147,8436 \\
\hline
\end{tabular}

$\mathrm{BC}$ - means the image before contrast, and AC - after contrast. The results of the mean precontrast differentiation indicate a much greater agreement in the first measurement in terms of angiogenesis assessment than in the measurement after contrast administration. At the same time, there was also an average lower number of pixels considered a significant indicator in the analysis of vessels in the I measurement. The mean absolute distance in the height of the histogram bars showed a significant differentiation, but the assessment of the average cost of transferring bars (EMD) was much higher.

A similar analysis was performed for the left and right side of the imaging. The results are presented in Table 2 . 
Table 2. Statistics for the mean values from the analysis of the histograms sides of the MRI (before and after contrast administration).

\begin{tabular}{|c|c|c|c|c|}
\hline & N important & Mean & Standard deviation & Coefficient of variation \\
\hline Med. LS BC & 48 & 440,89 & 102,9 & 23,3416 \\
\hline Var. LS BC & 48 & 28893,34 & 11975,8 & 41,4482 \\
\hline Med. RS BC & 48 & 521,46 & 221,7 & 42,5126 \\
\hline Var. RS BC & 48 & 56239,74 & 151337,0 & 269,0926 \\
\hline Med. LS AC & 48 & 458,47 & 107,4 & 23,4304 \\
\hline Var. LS AC & 48 & 31135,89 & 11400,9 & 36,6166 \\
\hline Med. RS AC & 48 & 541,48 & 223,0 & 41,1754 \\
\hline Var. RS AC & 48 & 56824,10 & 131366,2 & 231,1804 \\
\hline
\end{tabular}

In terms of the comparison of the right (RS) and left (LS) side before contrast administration, a higher average number of vessels is visible on the right side of the image in relation to the left side - which is also significantly different, measured by the standard deviation in this range. The variability of the standard deviation in both halves of imaging was different - for the right side it was almost $270 \%$ of the mean, and for the left side - less than $50 \%$ of the mean. Contrast administration did not change this characteristic significantly - the left side of the imaging still shows a lower number of vessels and less differentiation. The difference for both sides of the images after contrast administration is slightly smaller than in the image before contrast administration.

One of the measurement elements was the analysis of the image without the mask - the basic characteristics were also determined for them (Table 3). 
Table 3. Statistics for mean values from the analysis of histogram sides of MRI images (before and after contrast administration) without mask.

\begin{tabular}{|c|c|c|c|c|}
\hline & N important & Mean & Standard deviation & Coefficient of variation \\
\hline Med. LS BC & 48 & 454,77 & 114,39 & 25,1535 \\
\hline Var. LS BC & 48 & 16321,24 & 11126,39 & 68,1712 \\
\hline Med. RS BC 2 & 48 & 469,52 & 117,71 & 25,0697 \\
\hline Var. RS BC 2 & 48 & 16758,48 & 6619,42 & 39,4989 \\
\hline Med. LS AC 2 & 48 & 516,92 & 224,36 & 43,4040 \\
\hline Var. LS AC2 & 48 & 31722,39 & 72388,25 & 228,1929 \\
\hline Med. RS AC 2 & 48 & 537,50 & 234,03 & 43,5404 \\
\hline Var. RS AC 2 & 48 & 35593,68 & 81156,78 & 228,0090 \\
\hline
\end{tabular}

In the measurement without the mask before contrast administration, the results in the right and left-hand side were similar, the differentiation (mean of variance) in both groups was similar, and the disproportion to the mean measured by the coefficient of variation was much smaller. After administration of the contrast agent, an increase in the mean number of blood vessels is visible, and the variance in the number doubles. However, the equivalence of the coefficients of variation for the right and left variances was also observed.

In order to check the relationship between the number of microvessels and the histological type of the tumor, a correlation matrix was prepared. The results are presented in the following tables - for the image before contrasting, after administering the contrast, and with and without the mask. The results in imaging without the mask are presented in the table below (Table 4).

Table 4. Relationships between tumor type and imaging without the mask. Spearman's rank order correlation (tumors) removed in pairs. The determined correlation coefficients are significant with $\mathrm{p}$ $<0.05000 .{ }^{*} \mathrm{p}<0,05$

\begin{tabular}{|c|c|c|}
\hline & $\begin{array}{c}\text { Tumor malignancy according to WHO } \\
\text { grading }\end{array}$ & $\begin{array}{c}\text { Malignancy of the tumor } \\
\text { (malignant / benign) }\end{array}$ \\
\hline Med. BC & $-0,319676^{*}$ & $-0,222961$ \\
\hline Var. BC & $-0,090555$ & $-0,053818$ \\
\hline Med. AC & $-0,257859$ & $-0,170681$ \\
\hline Var. AC & $-0,047165$ & 0,019990 \\
\hline
\end{tabular}


One statistically significant rank correlation coefficient was obtained - the mean of the total number of vessels before contrast administration is higher in people with a lower tumor grade according to WHO. The relationship was significant, but its strength was average. The remaining relations, although opposite, are weak or slight and statistically insignificant.

The right and left sides of MRI images were also analyzed. Data for them are included in Table 5.

Table 5. Spearman's rank order correlation for MRI imaging sides and tumor type.

\begin{tabular}{|c|c|c|}
\hline & $\begin{array}{c}\text { Tumor malignancy according to WHO } \\
\text { grading }\end{array}$ & $\begin{array}{c}\text { Malignancy of the tumor } \\
\text { (malignant / benign) }\end{array}$ \\
\hline Med. LS BC & $-0,252788$ & $-0,189133$ \\
\hline Var. LS BC & $-0,144990$ & $-0,161455$ \\
\hline Med. RS BC & $-0,300573 *$ & $-0,232187$ \\
\hline Var. RS BC & $-0,092415$ & $-0,084572$ \\
\hline Med. LS AC & $-0,200607$ & $-0,146078$ \\
\hline Var. LS AC & $-0,085991$ & $-0,124551$ \\
\hline Med. RS AC & $-0,220950$ & $-0,176831$ \\
\hline Var. RS AC & $-0,099628$ & $-0,109174$ \\
\hline
\end{tabular}

In terms of the sides of the image, there was also one statistically significant relationship between the mean number of microvessels on the right side of the image before contrast administration and the tumor grade. This relationship was average, opposite and statistically significant. The other relations were of the same direction, but of lower strength and were statistically insignificant.

As the images without the mask were also analyzed, they were divided into pages - here they were also correlated with the tumor type - as presented in Table 6. 
Table 6. Spearman's rank order correlation of unmasked MRI sides and tumor type. ${ }^{*} \mathrm{p}<0,05$

\begin{tabular}{|c|c|c|}
\hline & $\begin{array}{c}\text { Tumor malignancy according to WHO } \\
\text { grading }\end{array}$ & $\begin{array}{c}\text { Malignancy of the tumor } \\
\text { (malignant / benign) }\end{array}$ \\
\hline Med. LS BC & $-0,344752^{*}$ & $-0,247564$ \\
\hline Var. LS BC & $-0,109320$ & $-0,059969$ \\
\hline Med. RS BC 2 & $-0,275497$ & $-0,186057$ \\
\hline Var. RS BC 2 & $-0,104981$ & $-0,072270$ \\
\hline Med. LS AC 2 & $-0,261297$ & $-0,164530$ \\
\hline Var. LS AC 2 & 0,014088 & 0,063044 \\
\hline Med. RS AC 2 & $-0,249069$ & $-0,167605$ \\
\hline Var. RS AC 2 & $-0,011890$ & 0,032291 \\
\hline
\end{tabular}

Table 6. Spearman's rank order correlation of unmasked MRI sides and tumor type. ${ }^{*} \mathrm{p}<0,05$

Imaging without the mask revealed a relationship with the tumor type for the right side of the image before contrast administration - so the relationship described above was preserved. The strength of this relationship was analogous to the previous ones (average), the direction of this relationship was also maintained.

Table 7. Correlation of Spearman's rank order in MDV index and tumor grade.

\begin{tabular}{|c|c|c|}
\hline & $\begin{array}{c}\text { Tumor malignancy according to WHO } \\
\text { grading }\end{array}$ & $\begin{array}{c}\text { Malignancy of the tumor } \\
\text { (malignant / benign) }\end{array}$ \\
\hline MVD in tumor tissue & $0,468790^{*}$ & $0,349558^{*}$ \\
\hline
\end{tabular}

The relationship between the MDV index in the tumor tissue and the tumor grade was found to be higher than in the case of imaging, indicating a consistent direction of the relationship of average strength. The greater the number of microvessels, the higher the degree of malignancy of the tumor. In the dichotomous division, the strength of the relationship decreases - which is caused by a smaller number of compared groups.

Despite the determination of the level of relationship, statistically significant differences were also searched for in the compared images according to the type of cancer. Their nature was estimated for all variables, but due to their length, only statistically significant distributions were included in the study.

The first statistically significant difference $(\mathrm{H}(3, \mathrm{~N}=48)=11.34556 \mathrm{p}=0.0100)$ was seen for the microvessel density in the tumor tissue. The above-mentioned correlation coefficient was of average strength, due to its non-linear nature - the study of differences revealed the nature of the relationship. 


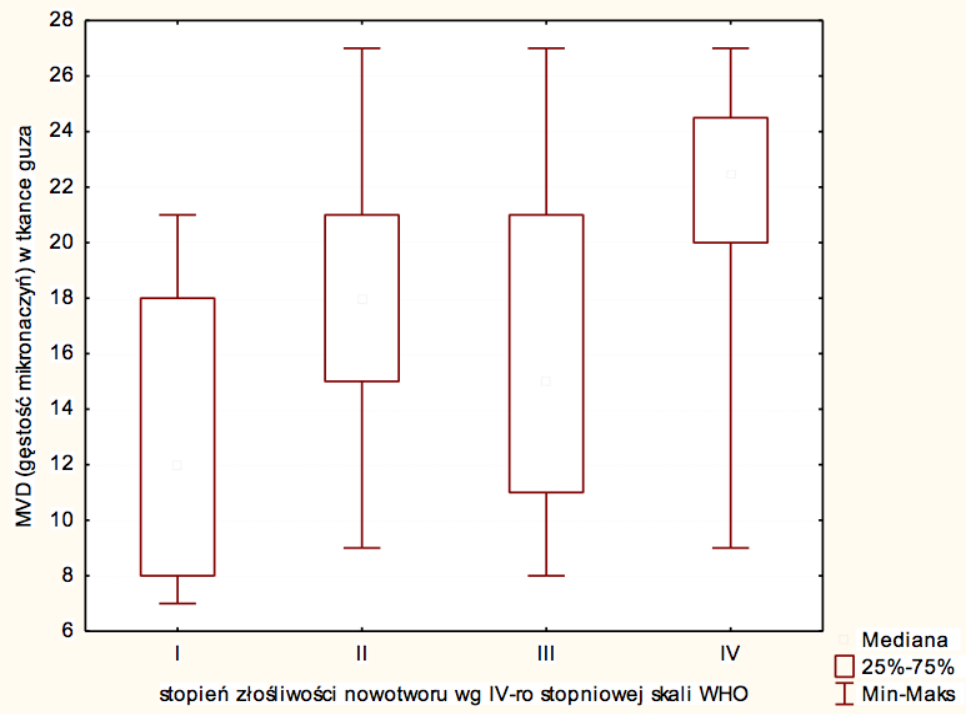

Figure 1. Differences in MDV distribution due to the malignancy of the tumor.

The presented graph clearly shows that the growth model is stepped - between II and III degrees in the WHO scale the relative balance of the number of microvessels is maintained, while a significant jump in the amount is visible between I and II and III and IV degrees of malignancy.

Similar relationships were observed for imaging microvessels and the type of tumor malignancy - but to a limited extent. The differences were significant for the mean number of vessels before contrast administration $(\mathrm{H}(3, \mathrm{~N}=48)=10.42578 \mathrm{p}=.0153)$, the nature of the differences is shown in Figure 2.

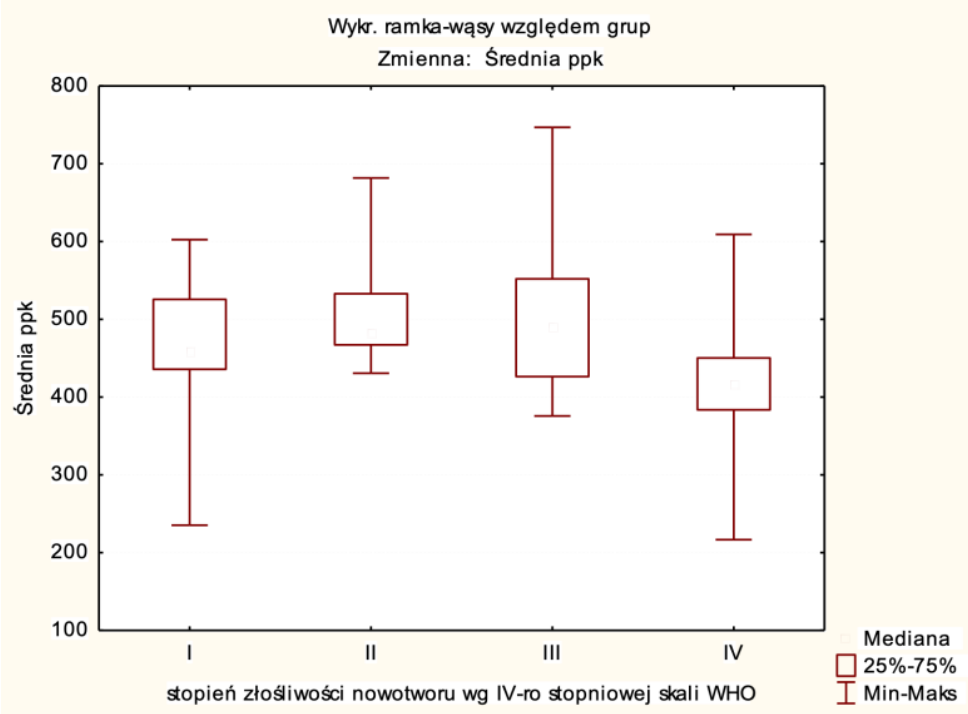

Figure 2. Differences in microvessel distribution before contrast administration due to malignancy of the tumor.

In the case of imaging before contrast administration, a high spread of results in grade I and IV is visible, and the number of vessels in these groups is usually lower than in the others. This image changes when contrast is applied. Again, the differences are statistically significant $(\mathrm{H}(3, \mathrm{~N}$ $=48)=7.796685 \mathrm{p}=.0490$ ), but of a different nature, as shown in Figure 3. 


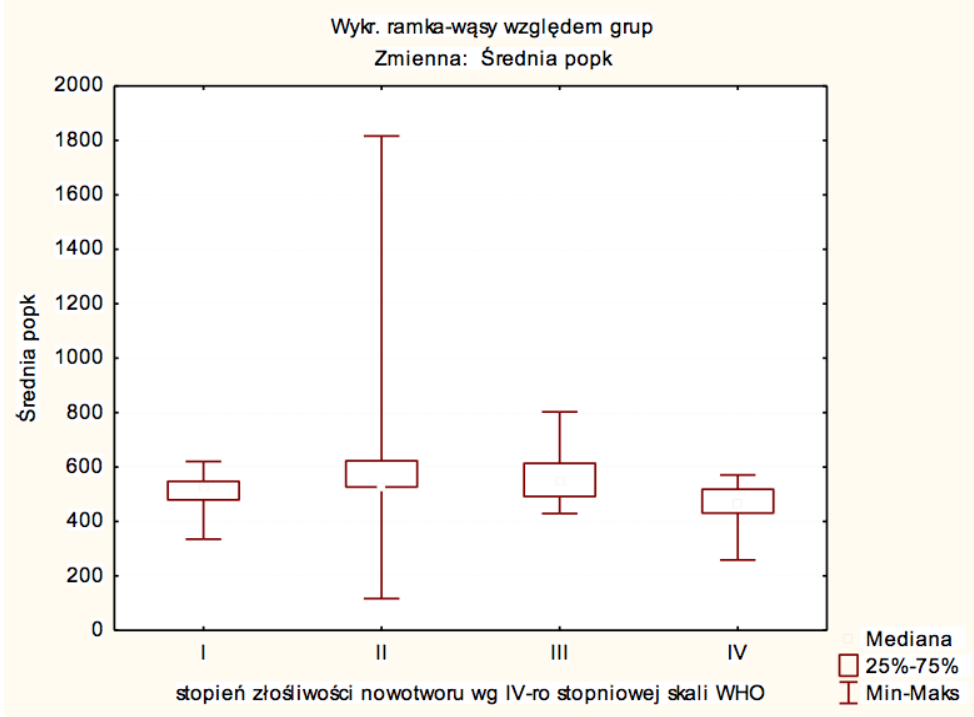

Figure 3. Differences in microvessel distribution after contrast administration due to the malignancy of the tumor.

The greatest differentiation is visible in the second degree of malignancy, but still, on average, the lowest number of vessels was observed for the I and IV degrees of malignancy. Examination of the sides of the image also revealed a difference - it was significant at the level of the statistical trend in the left side of the image before contrast $(\mathrm{H}(3, \mathrm{~N}=48)=7.145379 \mathrm{p}$ $=.0674)$. Its character is shown in Figure 4.

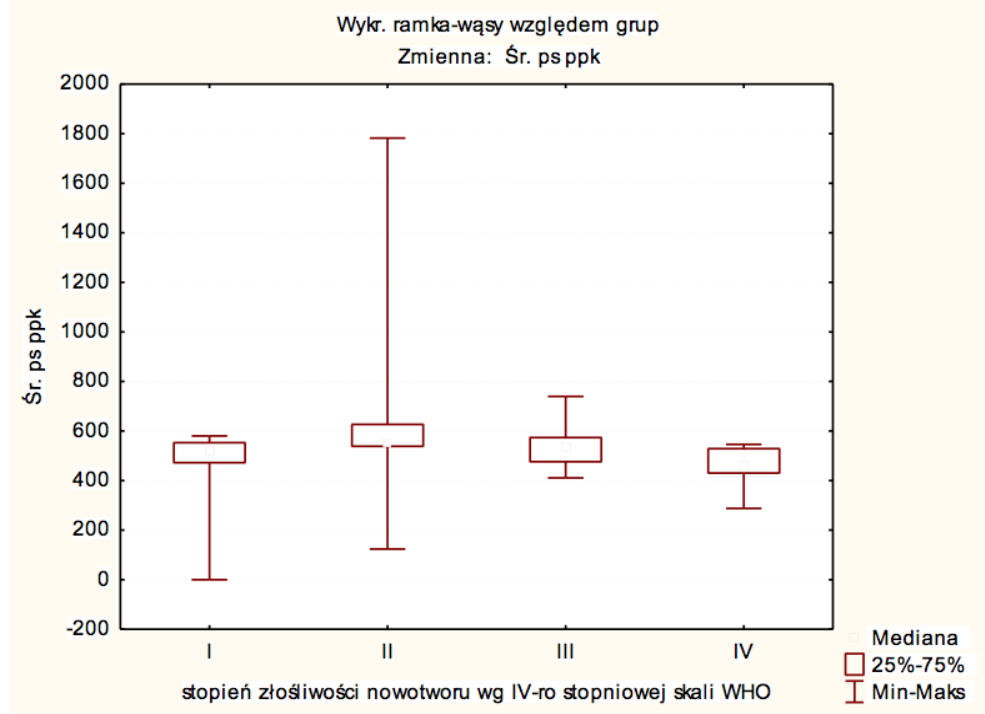

Figure 4. Differences in the distribution of microvessels in the left side of the image before contrast administration due to the malignancy of the tumor. 
The preservation of the average number of microvessels in the image also on the left side indicates an inverted U-shaped character, however, in this case, a disproportionately large range of results was revealed in the group defined as WHO grade I.

The analysis of the right part of the image also provided information about the differences this time they were statistically significant $(\mathrm{H}(3, \mathrm{~N}=48)=9.211293 \mathrm{p}=.0266)$. The behavior of the distribution of the average remained similar to the previous analysis, as shown in Figure

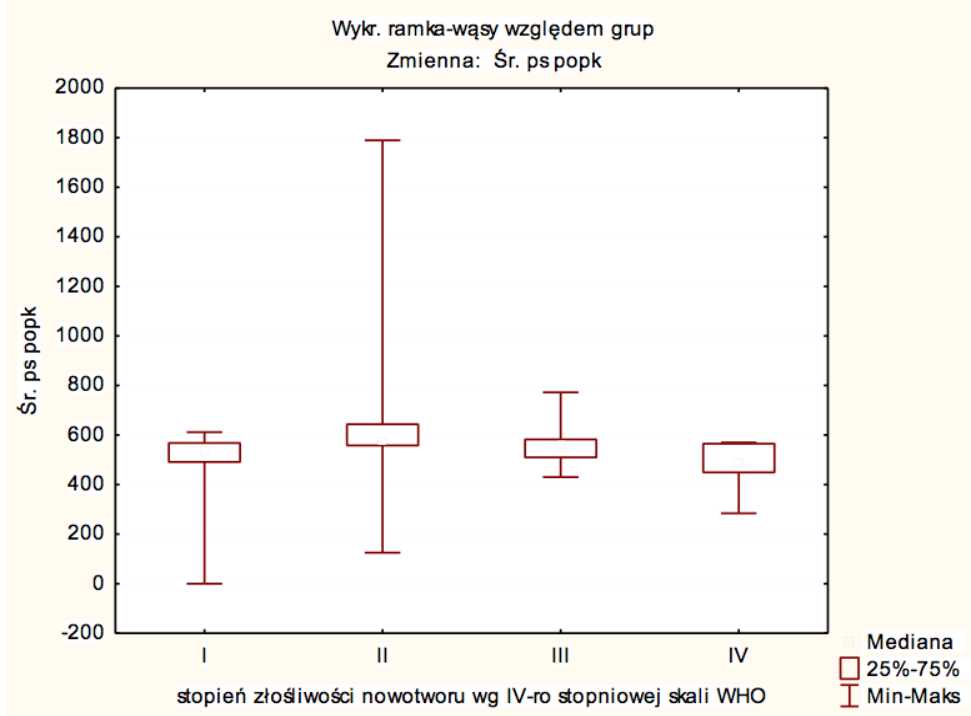

Figure 5. Differences in the distribution of microvessels on the right side of the image before contrast administration due to the malignancy of the tumor.

The intra-group differentiation in terms of the number of vessels was much greater in the second degree of malignancy than in the case of I. The remaining groups show a certain consistency of distribution, regardless of the imaging side.

Significant differences remained after administration of contrast in the range of sides. For the right side of the image, they were $(\mathrm{H}(3, \mathrm{~N}=48)=8.336835 \mathrm{p}=0.0395)$. The visualization of differences is presented in Figure 6.

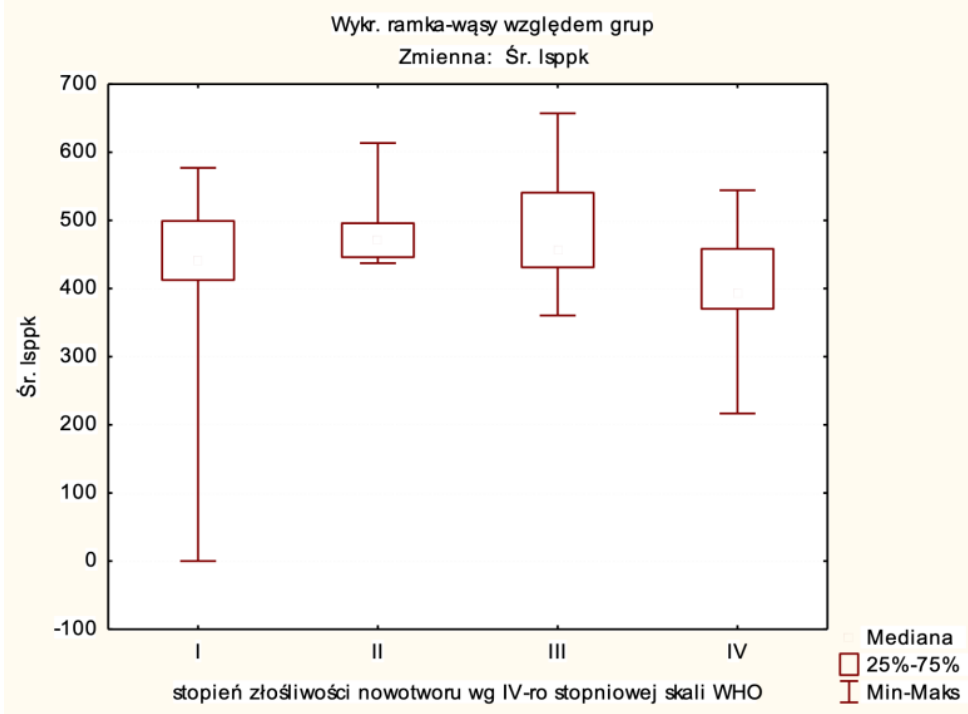

Figure 6. Differences in microvessel distribution on the right side of the image after contrast administration due to the malignancy of the tumor. 
In this case, the greatest differentiation remained in the second degree of malignancy, but in this group the average number of microvessels is visible. A large differentiation in the concentration of the results in the lower point is visible for group I, while in the others, such large variations are not visible.

In the case of the remaining parts of the images or the images of the mask, no statistically significant differences in the number of imaged tumor microvessels were found.

The analyzes presented above gave rise to the hypothesis that there is a relationship between the MVD index and the number of microvessels imaged with MRI, and the degree of malignancy and the type of tumor. This relationship is much stronger for the MDV index in tumor tissue than for imaging. At the same time, it was observed that the nature of the relationship is usually nonlinear - hence the correlation not exceeding the average strength. The relationship between the tumor type in the dichotomous malignant / benign division was not confirmed for imaging indicators.

In the last step, the results were analyzed for MVD in the tumor tissue by tumor type (Table $8)$.

Table 8. Descriptive statistics of MVD in tumor tissue by tumor type.

\begin{tabular}{|c|c|c|c|c|}
\hline $\begin{array}{c}\text { Tumor type (5 } \\
\text { general groups) }\end{array}$ & Mean & $\begin{array}{c}\text { Standard } \\
\text { deviation }\end{array}$ & Min & Max \\
\hline Metastatic tumor & 14,66667 & 8,500980 & 8,00000 & 27,00000 \\
\hline Meningioma & 13,66667 & 6,480741 & 7,00000 & 21,00000 \\
\hline Other & 18,33333 & 7,571878 & 13,00000 & 27,00000 \\
\hline Low Grade Glioma & 14,71429 & 4,855042 & 9,00000 & 21,00000 \\
\hline High Grade Glioma & 20,13043 & 5,479751 & 9,00000 & 27,00000 \\
\hline In total & 17,33333 & 6,537399 & 7,00000 & 27,00000 \\
\hline
\end{tabular}

Mean tissue microvessel density was highest in high-grade gliomas (20.13043) and other tumors (18.33333). The remaining tumors had a similar microvessel density (from 13.66667 to $14.71429)$ in total. The differentiation in metastatic tumors was by far the highest $(8,500980)$, while high similarity is characteristic of gliomas (LGG-4.855042, HGG-5,479751). The point ranges (min-max) are usually similar, although in HGG (9.00000-27.00000) and metastatic tumors (8.00000-27.00000) they are slightly larger. The average amount of MVD in the tissue, depending on the type of tumor, is presented in Figure 7. 


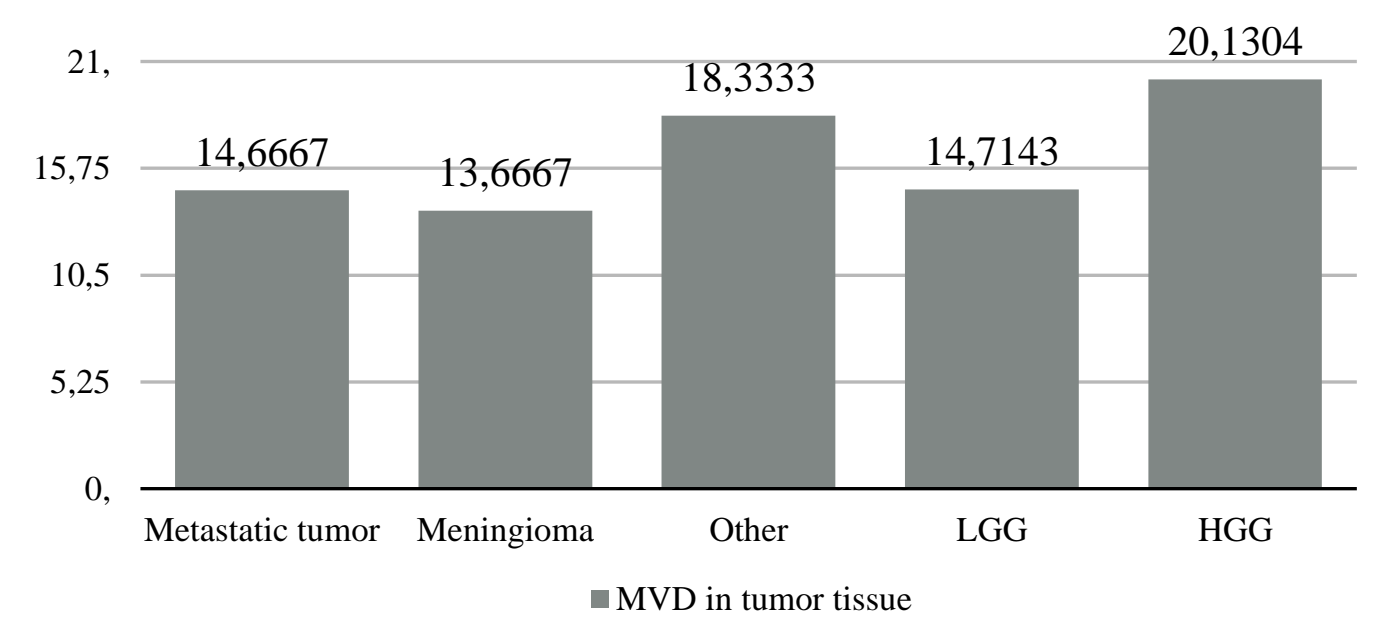

Figure 7. Mean amount of MVD in tumor tissue depending on the type of tumor.

\section{Discussion}

When examining the relationship between the number of tumor microvessels and its degree of malignancy, the MVD in the biopsy and the vessels imaged by MRI were assessed. The relationship between tumor grade and MVD was of average strength and indicated a consistent direction - the greater the number of microvessels, the higher the tumor grade. This relationship turned out to be much stronger than in the case of imaging. It was also found that the microvessel growth model is stepped - between the WHO II and III the relative number of microvessels is maintained, and a significant jump can be observed between I and II as well as III and IV degrees of malignancy. Similar relationships are also seen in the case of vessels imaged on MRI, both before and after the contrast administration. Interestingly, it was noticed that the number of microvessels is lower not only in grade I, but also in grade IV. This may be related to the areas of necrosis present in WHO grade IV tumors. Such results allowed for the hypothesis that there is a relationship between the MVD index and the number of microvessels imaged on MRI and the tumor grade. Lebelt [7] analyzed the vascularization of tumors of various degrees of malignancy on the basis of the assessment of microvascular proliferation (MVP) and microvessel density in their research on gliomas. The results showed the highest level of both MVP and MVD in glioblastoma, and the lowest in oligodnedroglioma, which clearly determines the existence of a significant correlation between tumor vasculature and its grade, and is therefore consistent with the results obtained in this study. Studying gliomas in adults and children, Birlik [8] also confirmed the existence of a relationship between MVD and the degree of tumor malignancy. He also found that in adult patients with MVD> 70, the survival time is significantly shorter than in those with MVD $<70$. A similar result was obtained by Lee [9] examining the usefulness of the MVD assessment as a prognostic factor for the prognosis of patients with astroglial tumors. There are also many reports of a strong positive correlation between the relative cerebral blood volume and the degree of histological malignancy of the tumor or MVD $[10,11,12]$. Each work found in the literature confirms the existence of the relations we found $[1,13,14]$. 


\section{Conclusions}

1. The highest mean density of MVD microvessels in tumor tissue can be observed in high-grade gliomas.

2. The high level of MVD expression in tumors of the central nervous system positively correlates with high tumor grade.

3. Determination of MVD expression level in tumor tissue is an additional prognostic factor in neoplasms of the central nervous system. The comparison of the results obtained with the routinely performed tests translates into a more accurate selection of treatment and assessment of the prognosis for survival.

\section{References}

1. Assimakopoulou M, Sotiropoulou-Bonikou G, Maraziotis T, Papadakis N, Varakis I. Microvessel density in brain tumors. Anticancer Res. 1997; 17: 4747-54.

2. Hlatky L, Hahnfeldt P, Folkman J. Clinical application of antiangiogenic therapy: microvessel density, what it does and doesn't tell us. J Natl Cancer Inst. 2002; 94: 883-93.

3. Mahzouni P, Mohammadizadeh F, Mougouei K, Moghaddam NA, Chehrei A, Mesbah A. Determining the relationship between "microvessel density" and different grades of astrocytoma based on immunohistochemistry for "factor VIII-related antigen" (von Willebrand factor) expression in tumor microvessel. Indian J Pathol Microbiol. 2010; 53(4): 605-10.

4. Kimura H, Takeuchi H, Koshimoto Y, et al. Perfusion imaging of meningioma by using continuous arterial spinlabeling: comparison with dynamic susceptibility-weighted contrast-enhanced MR images and histopathologic features. AJNR Am J Neuroradiol. 2006, 27(1):85-93.

5. Ferrara N, Kerbel RS. Angiogenesis as a therapeutic target. Nature, 2005, 438:967-74.

6. Folkman J. Angiogenesis in cancer, vascular, rheumatoid and other disease. Nat Med. 1995, 1:27-31.

7. Lebelt A, Dzięcioł J, Lemancewicz D, Zimnoch L, Czykier E. Angiogenesis in gliomas, Folia Histochemica et Cytobiologica 2008, 46(1):69-72.

8. Birlik B, Canda S, Ozer E. Tumour vascularity is of prognostic significance in adult, but not paediatric astrocytomas. Neuropathology and Applied Neurobiology 2006, 32, 532-8.

9. Lee SJ, Kim JH, Kim YM, et al. Perfusion MR imaging in gliomas: comparison with histologic tumor grade. Korean J Radiol 2001, 2:1-7.

10. Mahzouni P, Mohammadizadeh F, Mougouei K, Moghaddam NA, Chehrei A, Mesbah A. Determining the relationship between "microvessel density" and different grades of astrocytoma based on immunohistochemistry for "factor VIII-related antigen" (von Willebrand factor) expression in tumor microvessels. Indian J Pathol Microbiol. 2010, 53(4):605-10.

11. Nagar VA, Ye JR, Ng WH, Chan YH, Hui F, Lee CK, Lim CC. Diffusion-weighted MR imaging: diagnosing atypical or malignant meningiomas and detecting tumor de-differentiation, Am J Neuroradiol 2008, 29:1147-52.

12. McDonald DM, Choyke PL, Imaging of angiogenesis: from microscope to clinic. Nat Med. 2003, 9(6):713-25.

13. Hlatky L, Hahnfeldt P, Folkman J. Clinical application of antiangiogenic therapy: microvessel density, what it does and doesn't tell us. J Natl Cancer Inst. 2002; 94: 883-93.

14. Deb P, Boruah D, Dutta V. Morphometric study of microvessels in primary CNS tumors and its correlation with tumor types and grade. Microvascular Research [serial online] 2012; 84(1):34-43. 